\title{
Practices and attitudes of specialists in Forensic Medicine in the management of general health care needs of victims of sexual violence
}

\author{
Perera $\mathrm{J}^{{ }^{*}}$, Arambepola $\mathrm{C}^{2}$, Wijeratne $\mathrm{P}^{1}$ \\ ${ }^{1}$ Department of Forensic Medicine and Toxicology, ${ }^{2}$ Department of Community Medicine, Faculty of \\ Medicine, University of Colombo, Sri Lanka \\ *Corresponding author: Tel-0094-77-676-5983, e mail: pererajean32@yahoomail.com \\ MLSLL.Vol 1. No 3. December. pp 01-07
}

\begin{abstract}
Introduction

The emphasis of sexual assault forensic management (SAFM) in Sri Lanka has been mainly pertaining to collection of forensic evidence and as a referral point to other specialist services.

\section{Objectives}

To describe the practice and attitudes of Specialists in Forensic Medicine (SpFM) regarding the management of general health care needs in victims of sexual violence as part of sexual assault forensic management (SAFM) with a view to providing a victim friendly management.
\end{abstract}

\section{Methods}

A cross-sectional study was conducted to include all practicing (SpFM) in Sri Lanka. Victim centred practices as well as attitudes underlying their practice were assessed using a self-administered questionnaire.

\section{Results}

The response rate was $52.08 \%(n=25) .92 \%$ of the SpFM were males and had a mean age of 45.48 years $(S D=5.561)$. Many (48\%) felt that only those who appeared to be psychologically disturbed need to be referred to a counselor. The majority (72\%) never provided and $32 \%$ never prescribed emergency contraceptive pills (ECP) to victims. Most of them strongly disagreed that giving emergency contraception or reviewing the victim introduced victim bias.

\section{Conclusions}

Despite positive attitudes towards victim-friendly management, health care needs like pregnancy prevention and relieving psychological distress were not addressed adequately. Fear of being accused of victim bias was not a significant factor for not taking adequate steps. It could be due to inadequate awareness regarding new developments in victim friendly management and hesitancy to change existing practice during SAFM.

Key words: Sexual assault forensic examination, victim-friendly management, emergency contraception, attitudes, victim bias, health care needs 


\section{Introduction}

Sexual assault is an aggressive act motivated by power and control [1]. It has both long term and short term physical and psychological effects on the health and wellbeing of a victim. Thus, during sexual assault medical forensic management (SAFM), the health and welfare of patients become the foremost priority [1] that goes well beyond proving the case in a court of law [2]. As such, in addition to providing evidence for legal proceedings, forensic medical practitioners have a crucial responsibility to ensure that the health care needs of victims of sexual violence are addressed adequately in their clinical practice.

The "victim-friendly approach or management" advocated in many SAFM protocols recognizes that "every sexually assaulted victim deserves timely, compassionate, respectful and appropriate care"[3]. In many centres in the developed countries appropriate care in SAFM includes the treatment of injuries, prevention of unwanted pregnancies, prevention and treatment of sexually transmitted infections (STI), and the provision of psycho-social support and adequate rehabilitation programs $[3,4]$ in addition to the collection of forensic evidence. In these countries, victims are often referred to emergency medical units where all these services are available under one roof. However at times, lapses on addressing health care needs of these victims are reported from these countries as well. A survey done on emergency departments in Pennsylvania, USA revealed that $16 \%$ of the hospitals do not offer any emergency contraception nor counseling [5] while in USA on the whole, $42 \%$ of non-Catholic and 55\% Catholic hospitals neither dispense ECP nor provide a valid referral for ECP [6], thus causing lost therapeutic opportunities.

In Sri Lanka, the legal procedure when a victim lodges a police complaint regarding sexual violence is to subject her to medico-legal examination. Quite commonly, the first contact health care personnel for such examinations would be the Specialist in Forensic Medicine (SpFM).

In the absence of guidelines on providing victim-friendly SAFM, the SpFM are in the practice of engaging exclusively in the medicolegal aspect of care and referring the victims to relevant specialists for management of their general health care needs. However, there is no systematic approach to verify whether in fact all referred victims would access these clinical services.

Clinical guidelines ensure the provision of minimum care with acceptable standards and are proven to be effective universally $[1,3,7,8]$. However, when developing guidelines for providing victim-friendly SAFM, they should be pragmatic, based on the challenges faced by SpFM in addressing the general healthcare needs of victims as well as their own attitudes that may influence their practice. Authors particularly intended to find out whether fear of being accused of victim bias had affected the decision to look after the general health care needs. Therefore, in the absence of such evidence in Sri Lanka, we conducted a study that aimed at describing the practices and attitudes of SpFM in the management of general health care needs of victims of sexual violence.

\section{Methodology}

A descriptive cross-sectional study was conducted among all clinicians who were Board certified by the Postgraduate Institute of Medicine (PGIM) as Specialists in Forensic Medicine in Sri Lanka. Those who did not have more than six months experience as specialists and those who were not in active practice were excluded from the sample. Data on the attitudes and practices of SpFM with regards to psychological counseling, pregnancy and STI prevention, and review of victims was collected using a self-administered questionnaire prepared in English language. Guidelines for medico - legal care of victims of sexual violence by the World Health Organisation (WHO) ${ }^{1}$ were 
perused for developing the questionnaire. A five-point Likert scale ('strongly agree', 'agree', 'neutral', 'agree', 'strongly disagree') was used as responses to the questions on attitudes.

The level of victim friendly management during communication with the victim was assessed. The key phrases used were "speaking to victims in a nonjudgmental manner" , "encouraging the victim during history taking by appropriate body language", and using appropriate words like" hmm", "go on" with a scale ranging from 'always to never done'.

The questionnaire with an enclosed selfaddressed envelope and an information sheet about the objectives of this study were posted to all SpFM in the specialist register maintained at the PGIM.

They were requested to return the questionnaire within six weeks. Those who did not return the questionnaire at the end of six weeks were taken as not consenting to the study and thus taken as non-respondents.
Ethics clearance for the study was obtained from the Ethics review committee of the Faculty of Medicine, University of Colombo. SPSS statistical software was used for data analysis. Categorical data were described in proportions and quantitative data in mean and standard deviation (SD).

\section{Results}

Out of 48 SpFM Board certified by the PGIM, University of Colombo as at September 2012, 25 returned the completed questionnaires (response rate $=52.08 \%$ ).92\% of the participants were males and of the average age of 45.48 years $(S D=5.561)$. All except one $(96 \%)$ had examined more than 80 victims of sexual assault during their entire practice while the majority (57\%) had performed the last SAFM within the last week.

Most of the SpFM practiced speaking to a victim in a non-judgmental manner always or most of the time (84\%). (See Table 1.)

Table 1: Results concerning, following the formal routine in Communicating with victims $(n=25)$ (\%- Number of respondents as a percentage)

\begin{tabular}{|c|c|c|c|c|c|c|c|c|}
\hline \multirow[t]{3}{*}{ Form of routine } & \multicolumn{8}{|c|}{ Number of respondents } \\
\hline & \multicolumn{2}{|l|}{ Always } & \multirow{2}{*}{$\begin{array}{l}\text { Most } \\
\text { Time } \\
\text { Figure }\end{array}$} & \multirow{2}{*}{$\begin{array}{l}\text { of the } \\
\%\end{array}$} & \multicolumn{2}{|c|}{ Sometimes } & \multicolumn{2}{|l|}{ Never } \\
\hline & figure & $\%$ & & & figure & $\%$ & figure & $\%$ \\
\hline $\begin{array}{l}\text { Speaking to a victim in a non- } \\
\text { judgmental manner }\end{array}$ & 13 & $52 \%$ & 08 & $32 \%$ & 01 & $4 \%$ & 01 & $4 \%$ \\
\hline $\begin{array}{l}\text { Using appropriate gestures or non } \\
\text { verbal communication skills }\end{array}$ & 10 & $40 \%$ & 07 & $28 \%$ & 03 & $12 \%$ & 05 & $20 \%$ \\
\hline Using appropriate words & 10 & $40 \%$ & 08 & $32 \%$ & 02 & $8 \%$ & 05 & $20 \%$ \\
\hline
\end{tabular}

Only 10 (40\%) always used appropriate gestures. Two (8\%) used appropriate words only sometimes and $20 \%$ never did so. Out of the five who never used appropriate gestures or words reasons were given by three. One comment was "not aware of the importance of it', another, "gestures are not always reliable" and the other one "it is an inappropriate practice in medico-legal setting".
A statement was included in the questionnaire "Giving emergency contraception denotes partiality towards victims". Most of the participants $(80 \%)$ strongly disagreed with this statement. Furthermore, taking action to prevent pregnancy was considered as being 'extremely important' by $96 \%$ of responders. During SAFM, 80\% explained the risk of getting 
pregnant to victims 'always' or 'most of the time'.

However, a significant number (72\%) never provided emergency contraception to the victims and $32 \%$ never prescribed emergency contraceptive pills (ECP)(Table 02) One reason for not prescribing ECP was due to the referral made to a Gynecologist 'sometimes' (60\%) or 'most of the time' (20\%).
Reviewing the victim for late psychological complications was not a regular event, with $32 \%$ never reviewing and $16 \%$ rarely doing so (Table 02). Likewise, review for assessment of healing was not common, with 16(64\%) reviewing rarely or never. $72 \%$ considered that 'review of the victim was not extremely important. However, 68\% 'disagreed' or 'strongly disagreed' that reviewing the victim indicates victim bias.

Table 2: Prevention of pregnancy, referral for counseling and Post-examination reviews $(n=25)$ (\%- Number of respondents as a percentage)

\begin{tabular}{|c|c|c|c|c|c|c|c|c|c|c|}
\hline \multirow{3}{*}{ Form of routine } & \multicolumn{10}{|c|}{ Number of respondents } \\
\hline & \multicolumn{2}{|l|}{ Always } & \multicolumn{2}{|c|}{$\begin{array}{l}\text { Most of the } \\
\text { Time }\end{array}$} & \multicolumn{2}{|c|}{ Sometimes } & \multicolumn{2}{|l|}{ Rarely } & \multicolumn{2}{|l|}{ Never } \\
\hline & Figure & $\%$ & figure & $\%$ & figure & $\%$ & figure & $\%$ & figure & $\%$ \\
\hline $\begin{array}{ll}\text { Referral } & \text { for } \\
\text { psychological } & \\
\text { counseling } & \\
\end{array}$ & 09 & $(36 \%)$ & 06 & $(24 \%)$ & 04 & $(16 \%)$ & 01 & $(4 \%)$ & 05 & $20 \%$ \\
\hline Prescribing ECP & 02 & $(08 \%)$ & 04 & $(16 \%)$ & 09 & $(36 \%)$ & 02 & $(8 \%)$ & 08 & $32 \%$ \\
\hline $\begin{array}{l}\text { Review for late } \\
\text { psychological } \\
\text { complications }\end{array}$ & 01 & $4 \%$ & 04 & $16 \%$ & 08 & $32 \%$ & 04 & $16 \%$ & 08 & $32 \%$ \\
\hline $\begin{array}{lr}\text { Review } & \text { for } \\
\text { assessment } & \text { of } \\
\text { healing } & \end{array}$ & 01 & $04 \%$ & 03 & $12 \%$ & 05 & $20 \%$ & 07 & $28 \%$ & 09 & $36 \%$ \\
\hline
\end{tabular}

Only $60 \%$ referred the victim 'most of the time' or 'always' to a counselor. $48 \%$ either agreed or strongly agreed with the statement "only those who appear to have psychological disturbances should be counseled" (Chart 01).

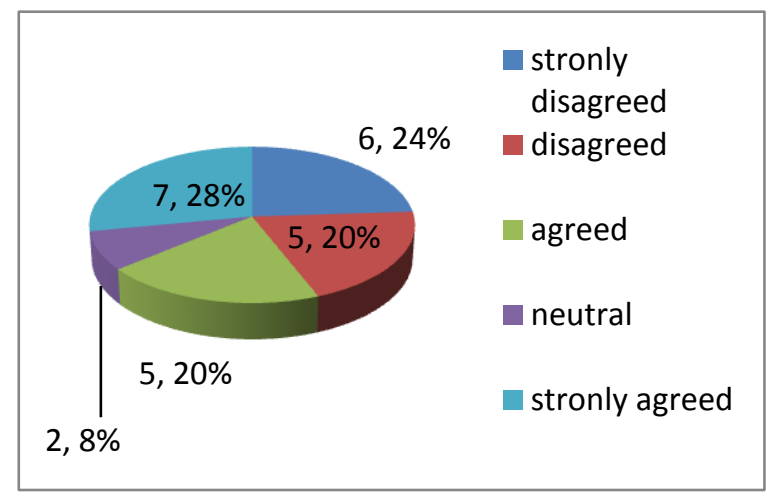

Information regarding risk of getting STI was given always or most of the time by $76 \%$ of respondents. STI prophylaxis (Table 03) was never prescribed by 23(92\%); lack of knowledge regarding STI prophylaxis being the reason given by $01 \mathrm{SpFM}$ for not prescribing. Others gave the reason as referral to STI clinic. STI prophylaxis was provided sometimes by $01(4 \%)$, most of the time by $01(4 \%)$ and never provided by the remaining $23(92 \%)$.

Unavailability of drugs for STI prophylaxis was given as the reason for not prescribing by 04 (16\%). Obtaining necessary samples for STI testing was never done by $21(84 \%)$, sometimes by $02(08 \%)$ and most of the time by $02(08 \%)$. However, all $25(100 \%)$ referred them to the STI 
clinic always or most of the time. 20(80\%) strongly agreed that it is their duty to prevent STI in sexual assault victims while 03(12\%) agreed with the view, but 02(8\%) strongly disagreed.

Table 03: Action taken to prevent STI $(n=25)(\%-$ Number of respondents as a percentage)

\begin{tabular}{|c|c|c|c|c|c|c|c|c|c|c|}
\hline \multirow{3}{*}{ Form of routine } & \multicolumn{10}{|c|}{ Number of respondents } \\
\hline & \multicolumn{2}{|l|}{ Always } & \multicolumn{2}{|c|}{ Most of the Time } & \multicolumn{2}{|c|}{ Sometimes } & \multicolumn{2}{|c|}{ Rarely } & \multicolumn{2}{|l|}{ Never } \\
\hline & Figure & $\%$ & figure & $\%$ & figure & $\%$ & figure & $\%$ & figure & $\%$ \\
\hline $\begin{array}{l}\text { Giving Information } \\
\text { regarding risk of } \\
\text { getting STI }\end{array}$ & 08 & $(32 \%)$ & 11 & $(44 \%)$ & 01 & $(04 \%)$ & 04 & $(16 \%)$ & 01 & $04 \%$ \\
\hline $\begin{array}{ll}\text { Prescribing } & \text { STI } \\
\text { prophylaxis } & \end{array}$ & - & - & 01 & $04 \%$ & 01 & $04 \%$ & - & - & 23 & $92 \%$ \\
\hline Referral to STI clinic & 13 & $52 \%$ & 12 & $48 \%$ & - & - & - & - & - & - \\
\hline
\end{tabular}

\section{Discussion}

The SpFM is in many instances the first contact health care professional for the sexual violence victim in Sri Lanka. Therefore, it is in the interest of the victim if SpFM addresses the general health care needs of the victim whenever possible or devise a mechanism to ensure they are addressed.

According to our study, SpFM seemed to have positive attitudes regarding prevention of pregnancy $(96 \%)$, with only one participant (4\%) dissenting, which indicates that they are much concerned about the welfare of the victim. Despite these positive attitudes, they preferred to entrust this responsibility to a Gynaecologist routinely even though there was no way of verifying whether they in fact obtained emergency contraception when indicated. Such dependence on the gynaecologist to prescribe or administer ECP can lead to secondary victimization due to long waits; consequent failure to comply can cause an unwanted pregnancy. This practice is unlikely to be due to the attitude that providing ECP indicates victim bias or partiality as $80 \%$ strongly disagreed with that view.
The role of therapy or psychological counseling for recovery from sexual violence is well established $[1,7,8]$. However, only $60 \%$ referred victims to a mental health professional or counselor most of the time or always. This may be in connection with the decision of $48 \%$ of the participants to refer only those victims who appear to be psychologically disturbed for counseling. This attitude appears risky as psychological effects of trauma may be more difficult to recognize [7] than physical effects especially to the doctor who has only a basic knowledge in psychiatry. When severely traumatized, victims can appear to be calm, indifferent, submissive, jocular, etc. [3], thus masking the real emotional disturbance. Furthermore, the late occurrence of mental health effects of sexual violence are well documented [7].

Reviewing the victim regularly is recommended in the WHO guidelines [1] and in many other protocols $[7,8]$. This is necessary for follow-up action for pregnancy and STI, to ascertain evidence of healing of injuries that could have medico-legal significance, to assess the emotional or mental status [1] and also for further relevant referral. However, according to 
our study, 32\% never reviewed the victim for psychological complications and $36 \%$ never reviewed for evidence of healing (Table 02). This practice reflects the attitude of the majority $(72 \%)$ that review is not extremely important. However, it can be concluded from this study that reluctance to review the victim was not directly connected with their fear of being accused of victimbiasas $68 \%$ of SpFM either disagreed or strongly disagreed with that view. It could probably be due to lack of awareness regarding the benefits and the global stand regarding review. However, 20\% have deviated from the traditional practice and have been conducting a review for psychological effects most of the time or always.

The role of a SpFM is to conduct the SAFM in a manner that initiates fast healing of the victim physically as well as mentally ${ }^{9}$. The fact that $84 \%$ treated the victims in a nonjudgmental manner, with $68 \%$ routinely using appropriate gestures - nonverbal communication and a significant percentage(72\%) often using appropriate words indicate that SpFM have endeavoured to create a victim friendly environment in SAFM. Using words like "please go on" and appropriate gestures such as nodding the head make victims comfortable and encourage them to reveal the complete story. However, $32 \%$ of the SpFM stated that they did not routinely use such gestures and 05(20\%) stated that they never used them. Judging from the comments, one reason for not using effective communication skills could be the traditional stand that medico-legal professionals have to appear impassive and without expression in order to appear impartial. On the contrary, an ideal SpFM should be sensitive to the victim's physical and psychological trauma while avoiding partisanship [4] and being impartial [1]. Another reason is the inadequate training in nonverbal communication skills as commented by one or even lack of awareness for its necessity in SAFM [9].

Conforming with standard traditional practice for SpFM in Sri Lanka 92\% never prescribed or provided STI prophylaxis but all took necessary action by referring them to the STI clinic either always or most of the time which is satisfactory. But it is interesting to note that 02 have broken away from the traditional practice and have been either prescribing or providing STI prophylaxis as advocated in many protocols.

\section{Limitations of the study}

The response rate was only $52.08 \%$, which was relatively satisfactory for a postal questionnaire.

\section{Conclusions}

In keeping with the current global thinking, the majority of SpFM possessed the attitude that attending to general health care needs such as prevention of pregnancy and STI, provision of counseling and victim friendly communication was important. However, many did not take adequate steps to apply it to practice except in the case of victim friendly communication. Fear of being accused of victim bias was not a significant factor for not taking adequate steps. One possibility is inadequate awareness of new global developments in SAFM. Another possibility is their attitude of not taking a different view from traditional practices, thereby being hesitant to change while confining themselves exclusively to taking care of only forensic management during SAFM.

\section{Recommendations}

Specialization courses in Forensic Medicine should include a mental health module and input in effective communication skills. Attitudes of SpFM described above should be taken into consideration when developing guidelines for the management of general health care needs of victims. Awareness programmes should be conducted for SpFM on the global changes regarding SAFM. Such programmes could contribute towards an attitudinal change towards victim-friendly SAFM. 


\section{References:}

1. Guidelines for medico - legal care of victims of sexual violence. Geneva, World Health Organization, 2003.

2. Nadesan K (2001)Rape: An Asian perspective. Journal of clinical forensic medicine, 8: $93-98$.

3. U. S. Department of Justice. A national protocol for sexual assault medical forensic examinations (adults/adolescents), U.S. Department of Justice, Office of Violence Against Women; 2004.

4. Cunningham N (2012) Sexual assault consultations: From high risk to high reliability. Journal of Forensic and Legal Medicine.19:pg 53-59

5. Harrison T. (2005) Availability of emergency contraception: a survey of hospital emergency department staff. Annals of Emergency Medicine, 46(2):105-110

6. Patel $A$, Simons $R$, Piotrowski ZH, Shulman L, Petraitis C. (2004)Under-use of emergency contraception for victims of sexual assault. International Journal of Fertility and Women's Medicine, 49(6):269-273.

7. Harris L, Freccero J, Sexual violence: medical and psychosocial support, Sexual violence and accountability project, Human Rights centre University of California, May 2011.
8. Clinical management of rape survivors: Developing protocols for use with refugees and internally displaced persons, InterAgency Lessons Learned Conference: Prevention and Response to Sexual and Gender-Based Violence in Refugee Situations, 2001, World Health Organisation, Geneva, Switzerland pg 25 28.

9. Perera JM, De Zoysa P. The need for effective communication skills in the medico-legal management of child sexual assault victims: observations from the Sri Lankan context. Sri Lanka Journal of forensic medicine science and law 2012;3(1) 16 - 19.

\section{Acknowledgements}

All specialists in Forensic Medicine who participated in the study, Dr. Subhani Poornima for assisting in data analysis, and formatting the paper.

\section{Contribution of authors}

Design and Supervision of the study - JP, CA

Data collection - JP, PW

Analysis of data - CA, JP, PW

Interpretation of results - JP, CA

Writing the manuscript - JP, CA, PW

Revising the manuscript $-C A, J P$ 